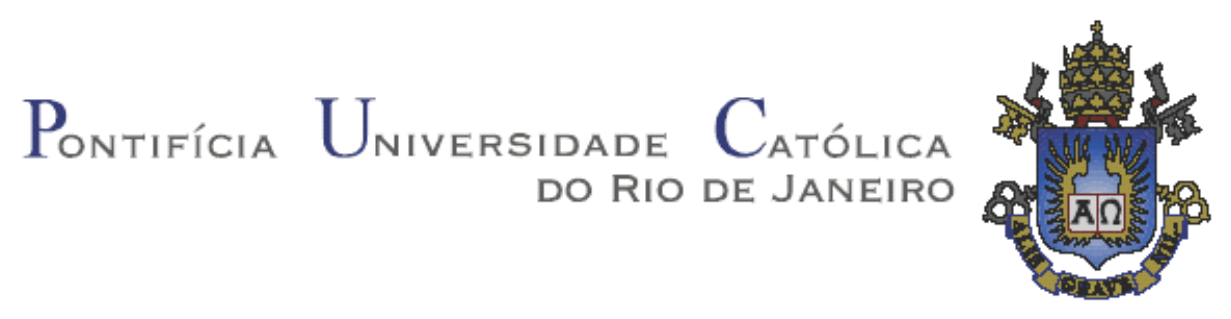

Mauro Artemio Carrión Pachás

\title{
Análise Limite com Otimizador de Grande Escala e Análise de Confiabilidade
}

Tese apresentada ao Programa de Pós-graduação em Engenharia Civil da PUC-Rio como requisito parcial para obtenção do título de Doutor em Engenharia Civil. Ênfase: Geotécnica

Orientador: Eurípedes do Amaral Vargas Júnior

Co-orientadores: Luíz Eloy Vaz

José Herskovits Norman

Rio de Janeiro, março de 2009 
Mauro Artemio Carrión Pachás

\section{Análise Limite com Otimizador de Grande Escala \\ e Análise de Confiabilidade}

Tese apresentada ao Programa de Pós-Graduação em Engenharia Civil da PUC-Rio como requisito parcial para obtenção do título de Doutor em Engenharia Civil. Aprovada pela Comissão Examinadora abaixo assinada.

Prof. Eurípedes do Amaral Vargas Júnior

Presidente/Orientador

Departamento de Engenharia Civil - PUC-Rio

Prof. Luiz Eloy Vaz

Co-Orientador-UFRJ

Prof. José Herskovits Norman

Co-Orientador-UFRJ

Prof. Luiz Fernando Marta

Departamento de Engenharia Civil - PUC-Rio

Prof. Ivan Menezes

PUC- Rio

Prof. Aldo Duran Farfán

UENF

Prof. Silvia Almeida

UFG

Prof. José Eugênio Leal

Coordenador Setorial do Centro Técnico Científico - PUC-Rio

Rio de Janeiro, março de 2009 
Todos os direitos reservados. É proibida a reprodução total ou parcial do trabalho sem autorização da universidade, do autor e do orientador.

\section{Mauro Artemio Carrión Pachás}

Graduou-se em Engenharia Civil na Universidade Nacional de Engenharia (UNI-PERU) em 1996. Trabalhou como pesquisador no Centro Peruano Japonês de Investigações Sísmicas e Mitigação de Desastres CISMID em Lima-Perú no período de 1997 a 2002. Estudou mestrado na PUC-Rio, em Engenharia Civil, na área de Geotecnia, no período 2002.2-2004.1. Ingressou no curso de doutorado na PUCRio no período 2004.2, atuando na linha de Pesquisa Numérica. Análise Limite com Otimizador de Grande Escala e Análise de Confiabilidade.

Ficha Catalográfica

Carrión Pachás, Mauro Artemio

Análise Limite com Otimizador de Grande Escala e Análise de Confiabilidade / Mauro Artemio Carrión Pachás; orientador: Eurípedes do Amaral Vargas Júnior; coorientadores: Luiz Eloy Vaz, José Herskovits Norman. 2009.

188 f. : il. ; $30 \mathrm{~cm}$

Tese (Doutorado em Engenharia Civil) - Pontifícia Universidade Católica do Rio de Janeiro, Rio de Janeiro, 2009.

Incluí referências bibliográficas.

1. Engenharia civil - Teses. 2. Análise limite. 3. Métdo de elementos finito. 4. Otimização. 5. Escoamento. 6. GEOLIMA. 7. Confiabilidade. 8. Função de falha. 9. FORM. I. Vargas Júnior, Eurípedes do Amaral. II. Vaz, Luiz Eloy. III. Norman, José Herskovits. IV. Pontifícia Universidade Católica do Rio de Janeiro. Departamento de Engenharia Civil. V. Título. 


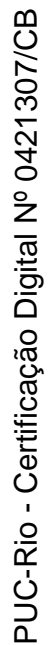

Para meus pais,

Juan Carrión e Marina Pachás,

pelo grande amor, confiança e exemplo.. 


\section{Agradecimentos}

Desejo expressar minha gratidão ao professor Eurípedes do Amaral Vargas Júnior, por ter-me convencido para fazer o curso de doutorado.

Aos professores Eurípedes do Amaral Vargas Júnior, Luíz Eloy Vaz e José Herskovits Norman pela orientação durante a realização deste trabalho.

Ao Professor Luiz Fernando Martha pela confiança e apoio.

Aos professores dos departamentos de Civil, Informática e Elétrica, pelos conhecimentos transmitidos em cada uma das disciplinas que cursei.

A meus pais, irmão, irmãs, meu primo Felix e a toda minha família, que sempre me apoiaram e incentivaram para a realização deste curso de doutorado.

À Mishel, pelo grande amor e compreensão, muito obrigado.

À Rita, secretária da pós-graduação, por sua atenção e disponibilidade.

À PUC-Rio, CAPES e CNPq pelos auxílios financeiros concedidos e ao TECGRAF/PUC-Rio pela oportunidade de poder trabalhar e estudar, sem a qual não teria sido possível realizar este estudo.

A todos os colegas do trabalho, do estudo e das peladas, muito obrigado pela convivência.

A Deus, porque sem a ajuda d'Ele nada acontece. 


\section{Resumo}

Pachás, Mauro Artemio Carrión; Vargas Júnior, Eurípedes do Amaral; Vaz, Luíz Eloy e Herskovits, José Norman. Análise Limite com Otimizador de Grande Escala e Análise de Confiabilidade. Rio de Janeiro, 2008. 188p. Tese de Doutorado - Departamento de Engenharia Civil, Pontifícia Universidade Católica do Rio de Janeiro.

O presente trabalho tem por objetivo desenvolver um otimizador eficiente de grande escala, que permita a aplicabilidade prática da Análise Limite Numérica pelo MEF, para resolver problemas reais da Engenharia Geotécnica. Para isto, foi desenvolvido um otimizador para o programa GEOLIMA (GEOtechnical LIMit Analysis) (Carrión, 2004) baseado no algoritmo de Pontos Interiores, computacionalmente mais eficiente que os otimizadores comerciais existentes. Pelo fato das propriedades do solo serem de natureza aleatória, a possibilidade de aplicar Análise de Confiabilidade com a Análise Limite pelo método FORM em problemas geotécnicos é pesquisada também. Sendo a grande vantagem do método FORM a possibilidade de se aplicar para funções de falha quaisquer e variáveis com distribuição quaisquer. Inicialmente, são apresentados os fundamentos da teoria de Análise Limite e sua formulação numérica pelo MEF (Método dos Elementos Finitos). A seguir, é investigada a possibilidade de se usar otimizadores comerciais para resolver o problema matemático resultante da aplicação de Análise Limite com o MEF e são descritos os fundamentos teóricos do otimizador implementado baseado no algoritmo de Pontos Interiores. Um resumo dos fundamentos teóricos da Análise de Confiabilidade é apresentado. É descrito o processo de cálculo pelo método FORM e dois exemplos de aplicação são realizados. Finalmente, análises de diferentes problemas resolvidos com o otimizador implementado são apresentados indicando o grande potencial da Análise Limite Numérica, na solução de problemas reais da Engenharia Geotécnica.

\section{Palavras-chave}

Análise limite; método de elementos finitos; otimização; função de escoamento; GEOLIMA; confiabilidade; função de falha; FORM. 


\section{Abstract}

Pachás, Mauro Artemio Carrión; Vargas Júnior, Eurípedes do Amaral; Vaz, Luíz Eloy e Herskovits, José Norman (Advisors). Limit Analysis with Large Scale Optimizer and Reliability Analysis. Rio de Janeiro, 2008. 188p. DrSc. These - Department of Civil Engineering, Pontifícia Universidade Católica do Rio de Janeiro.

This work has, as its main objective, the development of an efficient and large scale optimizer, that allows the practical application of Numerical Limit Analysis (NLA) with Finite Element Method (FEM) to solve real problems in Geotechnical Engineering. For that purpose, an optimizer was developed for GEOLIMA (GEOtechnical LIMit Analysis) program (Carrión, 2004), based on Interior Points algorithm, computationally more efficient than the existing commercial optimizers. Due to the fact that soils have random properties, the possibility to apply Reliability Analysis with Limit Analysis using the FORM method was also investigated. Initially, Limit Analysis theory was presented together with its numerical formulation using the FEM. In sequence, the use of commercial optimizers was investigated in order to solve the resulting mathematical problem. Subsequently, the theorical foundations of the developed optimizer, based on the Interior Points algorithm were described. A summary of Reliability Analysis was also presented together with a description of computational procedures using FORM and two examples were developed. Finally, analyses of different problems solved with developed optimizer were presented. The obtained results demonstrated the great potential of Numerical Limit Analysis (NLA), in the solution of real problems in Geotechnical Engineering.

\section{Keywords}

Limit analysis; finite element method; optimization; yield function; GEOLIMA; reliability; failure function; FORM. 


\section{Sumário}

1 INTRODUÇÃO

2 ANÁLISE LIMITE NUMÉRICA 23

2.1. Teoremas da Análise Limite 24

2.1.1. Campos de Tensões Estaticamente Admissíveis 24

2.1.2. Campos de Velocidades Cinematicamente Admissíveis 24

2.1.3. Teorema de Limite Inferior 25

2.1.4. Teorema de Limite Superior 25

2.2. Considerações na Análise Limite 25

2.2.1. Consideração de Plasticidade Perfeita 25

2.2.2. Considerações sobre Escoamento 26

2.2.3. Considerações sobre a Lei de Fluxo 28

2.3. Principio dos Trabalhos Virtuais $\quad 30$

2.4. Critérios de Escoamento 30

2.4.1. Critério de Mohr-Coulomb 31

2.4.2. Critério de Drucker-Prager 33

2.5. Formulação Numérica da Análise Limite pelo MEF 35

2.5.1. Condição de Equilíbrio 36

2.5.2. Condições de Contorno 38

2.5.3. Condição de Escoamento 38

2.5.4. Problema de Análise Limite 39

2.5.5. Elementos Finitos Implementados 39

3 SOLUÇÃO NUMÉRICA DA ANÁLISE LIMITE 41

3.1. Otimizadores Matemáticos Testados 42

3.1.1. Otimizador Lingo 43

3.1.2. Otimizador Minos 47

3.1.3. Otimizador Lancelot 50

3.1.4. Comparação de Desempenho de Otimizadores Testados 52

3.2. Otimizador Implementado 54 
3.2.1. Condições de Otimalidade

3.2.2. Algoritmo de Pontos Interiores 55

3.2.3. Inicialização 55

3.2.4. Direção de Busca 56

3.2.4.1. Técnica de deflexão 57

3.2.4.2. Técnica de relaxação-contração 58

3.2.4.3. Técnica Vetorial - Proposto 59

3.2.5. Comprimento de Passo 60

3.2.6. Atualização das Variáveis 61

3.2.7. Teste de Desempenho dos Algoritmos 62

3.2.8. Manipulação de sistemas lineares a serem resolvidos 63

3.2.8.1. Manipulação matricial global 64

3.2.8.2. Manipulação matricial por elementos 65

3.2.8.3. Solução direta sem manipulação - proposta 67

3.2.8.4. Teste de Desempenho das Manipulações 68

3.2.9. Resolvedores Implementados 75

3.2.9.1. Método dos gradientes conjugados (CG) 76

3.2.9.2. Teste de desempenho de resolvedores implementados 78

3.2.10. Resolvedor SAMG Testado 80

3.3. Melhora do desempenho 80

3.3.1. Tratamento de Matriz Esparsa 80

3.3.1.1. Formato CSR(Compressed Sparse Row) 80

3.3.1.2. Teste de desempenho de CG com tratamento da matriz esparsa81

3.3.2. Precondicionamento 82

3.3.2.1. Escala Diagonal (DS) 83

3.3.2.2. Escala Simétrica (SS) 83

3.3.2.3. Fatoração Incompleta de Cholesky (ICF) 84

3.3.2.4. Pré-condicionadores mistos - proposto 85

3.3.2.5. Teste de Desempenho de Pré-condicionadores 85

3.4. Teste de desempenho do Otimizador Implementado 87

3.4.1. Teste com problema em 2D 87

3.4.2. Teste com problema em 3D 90 
4 ANÁLISE DE CONFIABILIDADE COM ANÁLISE LIMITE 93

4.1. Conceitos Fundamentais da Análise de Confiabilidade 95

4.1.1. Incertezas 95

4.1.2. Função de Falha 95

4.1.3. Função Densidade de Probabilidade Conjunta 97

4.1.4. Probabilidade de Falha 99

4.1.5. Confiabilidade 100

4.1.6. Índice de Confiabilidade 101

4.1.7. Espaço Reduzido 103

4.1.8. Distribuição Normal Equivalente 105

4.2. Métodos de Cálculo 107

4.2.1. Método FORM (First-Order Reliability Method) 112

4.2.1.1. Transformação de Variáveis 112

4.2.1.2. Pesquisa de Ponto de Projeto 115

$\begin{array}{ll}\text { 4.2.1.3. Processo de Cálculo } & 117\end{array}$

4.3. Exemplos de Aplicação 118

$\begin{array}{ll}\text { 4.3.1. Talude 2D } & 118\end{array}$

4.3.2. Talude Confinado 3D 122

5 APLICAÇÕES 125

5.1. Análise 2D - Talude Infinito Homogêneo 126

5.2. Análise 2D - Talude Infinito Heterogêneo 134

5.3. Análise 2D - Talude com Percolação 142

5.4. Análise 2D - Barragem de Terra 146

5.5. Análise 3D - Talude Confinado 151

5.6. Análise 3D - Depósito de Rejeito 155

6 CONCLUSÕES E SUGESTÕES 161

6.1. Conclusões 161

6.2. Sugestões para Futuras Pesquisas 165

7 REFERÊNCIAS BIBLIOGRÁFICAS 166 
A.1. Variáveis Determinísticas e Aleatórias 171

A.2. Espaço Amostral, Evento e Valor Observado 171

A.3. Medidas de Tendência Central 171

A.3.1. Momento estatístico de ordem $m \quad 172$

A.3.2. Média aritmética 172

A.3.3. Características da média aritmética 173

A.4. Medidas de Dispersão da Variável Aleatória 173

A.4.1. Desvio 173

A.4.2. Momento estatístico central de ordem m 174

A.4.3. Desvio absoluto médio 174

A.4.4. Variância 174

A.4.5. Desvio padrão 175

A.4.6. Coeficiente de variação 176

A.5. Medidas de Correlação de Variáveis Aleatórias 177

A.5.1. Covariância 177

A.5.2. Coeficiente de correlação 178

A.6. Caracterização de Variáveis Aleatórias 180

A.6.1. Função Densidade de Probabilidade (PDF) 180

A.6.2. Função Distribuição de Probabilidade 182

A.6.3. Coeficiente de Inclinação de uma Distribuição 185

A.6.4. Coeficiente de Curtose 186

A.7. Esperança Matemática de uma Variável Aleatória 186 


\section{Lista de figuras}

Figura 2.1 - Relação tensão deformação para solo real e ideal (Chen, 1975). 26

Figura 2.2 - Superfície de escoamento no espaço de tensões principais. $\quad 27$

Figura 2.3 - Superfície de escoamento e vetor de deformação plástica. $\quad 29$

Figura 2.4 - Superfície de escoamento, critério de Mohr-Coulomb. 32

Figura 2.5 - Critério de escoamento de Mohr-Coulomb 2D. 33

Figura 2.6 - Critério de escoamento de Drucker \& Prager. 34

Figura 2.7 - Elemento finito: (a) quadrilateral (2D), (b) hexaédrico (3D). $\quad 40$

Figura 3.1 - Problema para teste de otimizadores. 42

Figura 3.2 - Malhas: (a) 28, (b) 64, (c) 126, (d) 225, (e) 360, (f) 500 e (g) 750 elementos. 43

Figura 3.3a - Variação da memória usada pelo otimizador LINGO. 45

Figura 3.3b - Variação de número de iterações do otimizador LINGO. 45

Figura 3.3c - Desempenho do otimizador LINGO. 46

Figura 3.3d - Variação do fator de colapso obtido com LINGO. 46

Figura 3.4a - Variação da memória usada pelo otimizador MINOS. 48

Figura 3.4b - variação de número de iterações do otimizador MINOS. 48

Figura 3.4c - Desempenho do otimizador MINOS. 49

Figura 3.4d - Variação do fator de colapso obtido com MINOS. 49

Figura 3.5a - Comparação de uso da memória entre Lingo e Minos 52

Figura 3.5b - Comparação de número de Iterações entre Lingo e Minos 52

Figura 3.5c - Comparação de desempenho entre Lingo e Minos 53

Figura 3.5d - Comparação de variação de fator de colapso, obtidos com Lingo e Minos. 53

Figura 3.6 - Técnica de deflexão. $\quad 57$

Figura 3.7 - Técnica de Relaxação-Contração. $\quad 59$

Figura 3.8- Técnica Vetorial - Proposta. $\quad 59$

$\begin{array}{ll}\text { Figura } 3.9 \text { - Comprimento de passo } s \text {. } & 61\end{array}$

Figura 3.10 - Atualização da variável $x$. $\quad 61$

Figura 3.11 Geometria do problema para teste de algoritmos (malha de 25 elementos). 62 
Figura 3.12 - Memória requerida pela manipulação matricial global.

Figura 3.13 - Tempo empregado na manipulação e solução do sistema. $\quad 70$

Figura 3.14 - Memória requerida para a solução do sistema. 71

Figura 3.15 - Tempo empregado na manipulação e solução do sistema. 71

Figura 3.16 - Memória requerida para a solução do sistema. 72

Figura 3.17 - Tempo empregado na solução do sistema. 73

$\begin{array}{ll}\text { Figura } 3.18 \text { - Memória usada pelas técnicas. } & 74\end{array}$

Figura 3.19 - Tempo empregado pelas técnicas. 75

Figura 3.20 - Algoritmo de Gradiente Conjugado pré-condicionado (Sandoval, 2006).

77

Figura 3.21 - Malhas: (a) 8, (b) 25, (c) 64, (d) 150, (e) 400 e (f) 676 elementos. 78

Figura 3.22 - Comparação do desempenho dos métodos implementados. $\quad 79$

Figura 3.23a - Armazenamento da matriz esparsa (SMAILBEGOVIC et all, 2006).

Figura 3.23b - Produto de uma matriz esparsa $A$ por um vetor $d$. 81

Figura 3.24 - Desempenho do método CG com tratamento da matriz esparsa CSR.

Figura 3.25 - Desempenho de CG com os pré-condicionadores implementados. 86

Figura 3.26 - Comparação de uso da memória pelos otimizadores. 88

Figura 3.27 - Comparação de número de iterações. 88

Figura 3.28 - Comparação do desempenho dos otimizadores. 89

Figura 3.29 - Variação de fator de colapso. 89

Figura 3.30 - Geometria da estrutura a ser analisada. 91

Figura 3.31 - Malha de elementos finitos (676 elementos e 945 nós). 91

Figura 3.32 - Mecanismo de colapso da estrutura obtido pelo programa

GEOLIMA 2.0. 92

Figura 3.33 - Mecanismo de ruptura obtido a partir de ensaios em modelo físico em escala reduzida (Sterpi,1996) 92

Figura 4.1 - Função de falha. 96

Figura 4.2 - Função densidade de probabilidade conjunta (Melchers, 2002). 99

Figura 4.3 - Probabilidade de falha. $\quad 100$

Figura 4.4 - Função densidade de probabilidade. 102

Figura 4.5 - Espaço original e espaço reduzido para uma variável. 104 
Figura 4.6 - Espaço original e reduzido para duas variáveis.

Figura 4.7 - Funções densidade de probabilidade PDF. 105

Figura 4.8 - Funções distribuição de probabilidade CDF. 106

Figura 4.9 - Variáveis em coordenadas reduzidas: função de falha não linear. 110

Figura 4.10 - Espaço original e reduzido para duas variáveis. 116

Figura 4.11 - Fluxograma da Análise de Confiabilidade pelo método FORM. 117

Figura 4.12 - Malha de elementos finitos 2D (25 elementos com 36 nós). 119

Figura 4.13 - Zonas de plastificação no MPP(Most Probable Point). 120

Figura 4.14 - Superfície de falha no MPP. 120

Figura 4.15 - Malha de elementos finitos 3D (27 elementos com 64 nós). 123

Figura 4.16 - Zonas de plastificação no MPP. 123

Figura 4.17 - Superfície de falha no MPP. 124

Figura 5.1 - Talude Infinito homogêneo. 127

Figura 5.2 - Malha de elementos finitos (500 elementos e 561 nós). 127

Figura 5.3(a) - Zonas de plastificação (talude com material coesivo). 128

Figura 5.3(b) - Vetor de velocidades (material coesivo). 128

Figura 5.3(c) - Superfície de falha (talude com material coesivo). 129

Figura 5.3(d) - Mecanismo de colapso (talude com material coesivo). 129

Figura 5.4(a) - Zonas de plastificação (talude com material com atrito). 130

Figura 5.4(b) - Vetor de veolicidades (talude com material com atrito). 130

Figura 5.4(c) - Superfície de falha (talude com material com atrito). 131

Figura 5.4(d) - Mecanismo de colapso (talude com material com atrito). 131

Figura 5.5(a) - Zonas de plastificação (talude com material maciço rochoso). 132

Figura 5.5(b) - Vetor de velocidades (talude com material maciço rochoso). 132

Figura 5.5(b) - Superfície de falha (talude com material maciço rochoso). 133

Figura 5.5(c) - Mecanismo de colapso (talude com material maciço rochoso). 133

Figura 5.6 - Talude infinito heterogêneo. 136

Figura 5.7(a) - Malha de elementos finitos 1 (100 elementos e 126 nós) . 136

Figura 5.7(b) - Zonas de plastificação (malha: 100 elementos e 126 nós). 137

Figura 5.7(c) - Superfície de falha (malha: 100 elementos e 126 nós). 137

Figura 5.8(a) - Malha de elementos finitos 2 (500 elementos e 561 nós). 138

Figura 5.8(b) - Zonas de plstificação (malha: 500 elementos e 561 nós). 138

Figura 5.8(c) - Superfície de falha (malha: 500 elementos e 561 nós). 139 
Figura 5.9(a) - Malha de elementos finitos 3 (4500 elementos e 4681 nós). 139

Figura 5.9(b) - Zonas de plastificação (malha: 4500 elementos e 4681 nós). 140

Figura 5.9(c) - Vetor de velocidades (malha: 4500 elementos e 4681 nós). 140

Figura 5.9(d) - Superfície de falha (malha: 4500 elementos e 4681 nós). 141

Figura 5.10 - Geometria de talude. 143

Figura 5.11 - Malha de elementos finitos (200 elementos e 231 nós). 143

Figura 5.12(a) - Zonas de plastificação, sem considerar percolação. 144

Figura 5.12(b) - Superfície de falha sem considerar percolação. 144

Figura 5.12(c) - Vetor de velocidades sem considerar percolação. 144

Figura 5.13(a) - Zonas de plastificação, considerando percolação. 145

Figura 5.13(b) - Superfície de falha, considerando percolação. 145

Figura 5.13(c) - Vetor de velocidades, considerando percolação. 145

Figura 5.14 - Plano em planta do projeto (CISMID, 2002). 147

Figura 5.15 - Secção 3-3 Barragem de Terra (CISMID, 2002). 148

Figura 5.16 - Malha de elementos finitos (624 elementos e 689 nós). 148

Figura 5.17(a) - Zonas de plastificação. 149

Figura 5.17(b) - Superfície de falha. $\quad 149$

Figura 5.17(c) - Vetores de velocidade. 149

Figura 5.18(a) - Zonas de plastificação. $\quad 150$

Figura 5.18(b) - Superfície de falha. 150

$\begin{array}{ll}\text { Figura 5.18(c) - Vetor de velocidade. } & 150\end{array}$

Figura 5.19 - Malha de elementos finitos (8000 elementos e 9261 nós). 152

Figura 5.20(a) - Zonas que plastificam. 152

Figura 5.20(b) - Vetor de veolocidades. 153

Figura 5.20(c) - Distribuição de campo de velocidades. 153

Figura 5.20(d) - Distribuição de campo de velocidades na seção longitudinal. 154

Figura 5.20 (e) - Mecanismo de colapso do talude. 154

Figura 5.21 - Topografia e limite do material de rejeito (CISMID, 1998). 156

Figura 5.22 - Modelo 3D da bacia sem material de rejeito. 157

Figura 5.23 - Material de rejeito. 158

Figura 5.24 - Malha de elementos finitos (2000 elementos e 2541 nós). 158

Figura 5.25(a) - Zonas de plastificação. 159

Figura 5.25(b) - Zonas de plastificação na seção longitudinal. 159 
Figura 5.25(c) -Vetor de velocidades. 159

Figura 5.25(d) - Distribuição de velocidades. 160

Figura 5.25(e) - Distribuição de velocidades na seção longitudinal. 160

Figura A.1 - Interpretação gráfica de coeficiente de correlação. 179

Figura A.2 - Função densidade de probabilidade normal. 182

Figura A.3 - Função distribuição de probabilidade normal. 183

Figura A.4 - (a) Função de variável aleatória; (b) Função de ponderação; (c) Esperança matemática da função de variável aleatória. 188 


\section{Lista de tabelas}

Tabela 3.1 - Testes realizados com o programa Lingo. 44

Tabela 3.2 - Testes realizadas com o otimizador Minos. 47

Tabela 3.3 - Comparação da eficiência entre Lancelot e Minos (Bongartz et all, 1997).

Tabela 3.4 - Desempenho dos algoritmos implementados 63

Tabela 3.5 - Teste da manipulação matricial global. $\quad 69$

Tabela 3.6 - Teste da manipulação matricial por elementos. 70

Tabela 3.7 - Teste da solução direta sem manipulação. 72

Tabela 3.8 - Comparação de resultados com as três técnicas de solução. $\quad 74$

Tabela 3.9 - Comparação do desempenho dos métodos implementados. $\quad 79$

Tabela 3.10 - Desempenho dos métodos com o tratamento da matriz esparsa. 82

Tabela 3.11 - Teste de pré-condicionadores implementados. 86

Tabela 3.12 - Resultados do teste em 2D do otimizador implementado. 87

Tabela 4.1 - Índice de confiabilidade e probabilidade de falha. 103

Tabela 4.2 - Coeficiente de correlação equivalente. 113

Tabela 5.1 - Propriedades de materiais e resultados da Análise Limite. 126

Tabela 5.2 - Problema de otimização e resultados da Análise Limite. 135

Tabela 5.3 - Propriedades de materiais e resultados da Análise Limite. 143

Tabela 5.4 - Propriedades dos materiais (CISMID, 2002). 148

Tabela 5.4 - Resultados da Análise Limite. 148

Tabela 5.5 - Propriedades dos materiais (CISMID, 1998). 157

Tabela A.1 - Grão de dependência das variáveis aleatórias. 179

Tabela A.2 - Funções densidade probabilidade (PDF) e de distribuição de probabilidade (CDF) ( Lopes, 2007). 184 


\section{Lista de Símbolos}

\section{Na Análise Limite ( $A L)$}

$F\left(\sigma_{i j}\right) \quad$ Função de escoamento em termos de tensões

$\sigma_{i j} \quad$ Campo de tensões

$I_{1} \quad$ Primeiro invariante de tensor de tensões

$J_{2} \quad$ Segundo invariante desviador

$J_{3} \quad$ Terceiro invariante desviador

$\dot{\varepsilon}_{i j} \quad$ Velocidade de deformação total

$\dot{\varepsilon}_{i j}^{e} \quad$ Velocidade de deformação elástica

$\dot{\varepsilon}_{i j}^{p} \quad$ Velocidade de deformação plástica

$\dot{\lambda} \quad$ Fator de proporcionalidade (escalar).

$\sigma_{1} \quad$ Tensão principal maior

$\sigma_{2} \quad$ Tensão principal intermédia

$\sigma_{3} \quad$ Tensão principal menor

$\theta \quad$ Ângulo de Lode

$\phi \quad$ Ângulo de atrito do material

C Coesão do material

$k, \alpha \quad$ Parâmetros do material (critério de Drucker-Prager)

$c_{0} \quad$ Resistência à compressão uniaxial

\section{No MEF}

$N_{i} \quad$ Função de interpolação

$r, s, t \quad$ Coordenadas locais

$r_{i}, s_{i}, t_{i} \quad$ Coordenadas locais nodais

$\sigma \quad$ Vetor campo de tensões

$\hat{\sigma} \quad$ Vetor campo de tensões nodais

$\dot{u} \quad$ Vetor campo de velocidades

$\hat{\dot{u}} \quad$ Vetor campo de velocidades nodais 
$\dot{\varepsilon} \quad$ Vetor campo de velocidades de deformação

$F_{0} \quad$ Força de volume inicial

$T_{0} \quad$ Força de superfície inicial

a Fator de colapso

$\boldsymbol{B} \quad$ Matriz de equilíbrio

b Vetor de carregamentos nodais

\section{Otimização}

$\begin{array}{ll}\boldsymbol{x} & \text { Vetor das variáveis } \\ \lambda, \boldsymbol{\mu} & \text { Multiplicadores de Lagrange } \\ n & \text { Número de variáveis } \\ m & \text { Número de restrições de desigualdade } \\ p & \text { Número de restrições de igualdade } \\ f(\alpha) & \text { Função objetivo } \\ \boldsymbol{g}(\boldsymbol{x}) & \text { Restrições de desigualdade } \\ \boldsymbol{G} & \text { Matriz de escoamento (diagonal) } \\ \boldsymbol{S} & \text { Matriz gradiente } \\ \boldsymbol{H} & \text { Matriz Hessiana } \\ \theta & \text { Ângulo de deflexão }\end{array}$

\section{Confiabilidade}

$X_{i} \quad$ Variável aleatória i

${ }^{j} x_{i} \quad j$-ésimo valor observado da variável aleatória $X_{i}$

${ }^{s} \bar{X}_{i}^{m} \quad$ Momento estatístico de ordem $m$ da amostra

${ }^{s} \bar{X}_{i} \quad$ Média da amostra da variável aleatória $X_{i}$

${ }^{s} X_{i}^{m} \quad$ Momento estatístico central de ordem $m$ da amostra

${ }^{s} X_{i} \quad$ Desvio absoluto médio da amostra

$\operatorname{Var}\left({ }^{s} X_{i}\right) \quad$ Variância da variável aleatória $X_{i}$

$\sigma_{x_{i}} \quad$ Desvio padrão da variável aleatória $X_{i}$

$\sigma \quad$ Matriz desvio padrão

$\delta_{x_{i}} \quad$ Coeficiente de variação da variável aleatória $X_{i}$ 
$\operatorname{Cov}\left(X_{1}, X_{2}\right) \quad$ Covariância das variáveis aleatórias $X_{1}$ e $X_{2}$

$\boldsymbol{S} \quad$ Matriz covariância

$\rho_{x_{j}, x_{k}} \quad$ Coeficiente de correlação das variáveis aleatórias $X_{j}$ e $X_{k}$

$\rho \quad$ Matriz coeficiente de correlação

$p(x) \quad$ Função densidade de probabilidade

$P\left(x_{a}\right) \quad$ Função distribuição de probabilidade

$\operatorname{Pr}\left[X \leq x_{a}\right]$ Probabilidade de que a variável $X$ seja menor ou igual a $x_{a}$

$X \quad$ Vetor de variáveis aleatórias

$F(X) \quad$ Função de falha

$\bar{F} \quad$ Média da função de falha

$\sigma_{F} \quad$ Desvio padrão da função de falha

$P_{f} \quad$ Probabilidade de falha

C Confiabilidade

$\beta \quad$ Índice de confiabilidade

$\boldsymbol{Y} \quad$ Variável aleatória reduzida

$\phi \quad$ Função distribuição de probabilidade normal padrão

$\Phi \quad$ Função cumulativa normal padrão

J Jacobiano

$\boldsymbol{L} \quad$ Matriz triangular inferior

$\nabla \quad$ Operador diferencial

$y^{*} \quad$ Ponto de pesquisa 